International Mathematical Forum, Vol. 9, 2014, no. 32, 1581 - 1593

HIKARI Ltd, www.m-hikari.com

http://dx.doi.org/10.12988/imf.2014.49168

\title{
A Toy Model for Hostility Between Two Populations in Dependency on Their Internal Frustration
}

\author{
Thomas Wieder \\ https://sites.google.com/site/twieder21stcenturyad/
}

Copyright (C) 2014 Thomas Wieder. This is an open access article distributed under the Creative Commons Attribution License, which permits unrestricted use, distribution, and reproduction in any medium, provided the original work is properly cited.

\begin{abstract}
Hostility between two populations $n$ and $m$ is described in terms of a first-order differential equation system for the population sizes $n(t)$ and $m(t)$ over time $t$. Each population is subdivided into two subpopulations 'Doves' and 'Hawks'. Hawks represent the strategy aggression against the other population. The number of hawks which actually exert aggression depends on the overall frustration within their population. Conversely, aggression causes the conversion from doves to hawks in the attacked population. Thus, a system of flows among the subpopulation is established. The actual behaviour of $n(t)$ and $m(t)$ over time $t$ depends on the coefficients chosen for the differential system and in particular on the temporal development of the frustration parameters. No calculation or simulation of actual population sizes is intended. The only goal of the paper is to establish a model which describes an never ending conflict between both populations caused by internal frustrations.
\end{abstract}

Mathematics Subject Classification: 91C99

Keywords: population dynamics, social strategies

\section{Introduction}

Consider two human populations $n$ and $m$. Both populations grow over time $t$ by reproduction. Furthermore, $n$ and $m$ exert violent actions against each other and therefore mutually cause losses to the other population. Thus, we 
are confronted with time-dependent functions $n=n(t)$ and $m=m(t)$. Our interest is not so much in the prediction of $n(t)$ and $m(t)$. Rather, we want to understand why such conflicts persist seemingly forever.

The kinematics of a long-lasting conflict is all to well known. Each party accuses the other one of something which in turn justifies the own doing. However, in the present model we do not model explicitly this kinematics. Our idea is that all this arguing and what allegedly has happened in the past is of secondary nature. The driving force for hostility of $m$ against $n$ is the internal frustration $\left(1-u_{m}(t)\right)$ of $m$ and conversely $n$ is hostile against $m$ because of the internal frustration $\left(1-u_{n}(t)\right)$.

How can we define the contentedness $u_{n}(t)$ of $n$ (and likewise $u_{m}(t)$ ) in sociological terms on the one hand and in quantitative terms on the other hand? Here we will abstain from a sociological discussion. Furthermore, we will use extremely simple, arbitrarily chosen expressions for $u_{n}(t), u_{m}(t)$. Our only goal is to introduce contentedness/frustration as a mechanism which subdivides a population into irenic and aggressive subpopulations. The aggressive subpopulation will apply violence to the other population in any case.

The present model accounts for the resulting interplay between both populations $n$ and $m$. If $m$ attacks $n$, then this aggression is perceived by $n$. In terms of the model, this perception leads to a flow from the irenic subpopulation of $n$ to the aggressive subpopulation of $n$. This flow is the stronger the stronger the frustration $\left(1-u_{n}(t)\right)$ is. The same mechanism applies to aggression from $n$ against $m$. Thus, the circle is closed, the conflict persists.

In section 4 we will discuss shortly the sense of such a toy model. At first however, we will outline the model in the next section.

\section{Model}

Consider a population $n$ with $n(t)$ members at time $t$. In general, $n$ is composed of certain subpopulations where each subpopulation represents a strategy $\mathcal{S}$ for the interaction with another given population $m=m(t)$. Hereby the strategy $\mathcal{S}_{m n}$ applied by population $n$ against population $m$ is defined by its quantitative impact on $m(t)$. That is, $\mathcal{S}_{m n}$ is expressed by a differential term depending on $n(t)$ in the differential equation for the growth $d m(t) / d t$ of $m(t)$ with time $t$. In the same way, $m$ may exert $\mathcal{S}_{n m}$ against $n$. We will consider two strategies only. Let $\mathcal{D}$ denote the strategy of non-aggressive behaviour (with $D$ for 'Dove'). Furthermore, let $\mathcal{H}$ denote the strategy of aggressive behaviour (with $H$ for 'Hawk'). We then imagine $n$ and $m$ to be composed of two subpopulations $n_{D}$ ('Doves') and $n_{H}$ ('Hawks') with

$$
\begin{aligned}
n(t) & =n_{D}(t)+n_{H}(t) \\
m(t) & =m_{D}(t)+m_{H}(t) .
\end{aligned}
$$


Furthermore, we introduce the notion of contentedness $u_{n}=u_{n}(t)$ as a quantitative feature of $n$ (and similarly for $m$ ). Each member of $n$ has a certain individual contentedness and there will be a certain distribution of individual contentedness within $n$. We neither model explicitly $u_{n}$ as a psychological quantity nor model the distribution of $u_{n}(t)$ in $n$. We just define $u_{n}(t)$ quantitatively as a fraction according to

$$
\begin{aligned}
n_{D l}(t) & =u_{n}(t) n_{D}(t) \\
n_{D h}(t) & =\left(1-u_{n}(t)\right) n_{D}(t) \\
n_{D}(t) & =n_{D l}(t)+n_{D h}(t) .
\end{aligned}
$$

In this manner $n_{D l}(t)$ is the fraction of content $=$ non-frustrated doves and $n_{D h}(t)$ is the fraction of frustrated doves. Similar equations apply to the aggressive subpopulation $n_{H}$, thus $n_{H}(t)=n_{H l}(t)+n_{H h}(t)$. Consequently, $n_{H l}(t)$ is the fraction of non-frustrated hawks and $n_{H h}(t)$ is the fraction of frustrated hawks. In total we have

$$
\begin{aligned}
n(t) & =n_{D l}(t)+n_{D h}(t)+n_{H l}(t)+n_{H h}(t) \\
m(t) & =m_{D l}(t)+m_{D h}(t)+m_{H l}(t)+m_{H h}(t) .
\end{aligned}
$$

Figure 1 exhibits graphically the relations (6).

Now, $n$ and $m$ grow over time. We express the growth rates $d n(t) / d t$ of $n$ and $d m(t) / d t$ of $m$ by a differential equation system which will be outlined now. Two classes of growth will occur. Internal growth appears among two subpopulations of a given population. Actually internal growth is just a transition from one subpopulation to another subpopulation. Consequentially, internal growth does not change the total number $n(t)$. In this model, the internal growth is governed by the dimensionless parameter $u_{n}(t)$. External growth on the contrary affects $n(t)$. New members enter $n$ by reproduction ('birth') and other members leave $n$ by annihilation ('death'). In the following, we often will speak of transitions, reproduction and annihilation as fluxes. Let us now introduce the corresponding quantitative expressions for these fluxes.

Frustrated members of $n_{D}$ belong to $n_{D h}(t)=\left(1-u_{n}(t)\right) n_{D}(t)$ and these members are susceptible to a transition from $n_{D h}$ to $n_{H h}$. The interaction mechanism among $n$ and $m$ comes into play here. If the probability of an aggressive action by a member of $m_{H}$ against $n$ is given by $W_{n m}$, then $W_{n m} m_{H}(t)$ is the number of aggressive actions of $m_{H}$ against $n$ at time $t$. Our model allows for a reaction to an action. Each aggression against $n$ raises the transition from $n_{D}(t)$ to $n_{H}(t)$ by the amount $C_{n, H D} W_{n m}$ (1$\left.u_{n}(t)\right) n_{D}(t)$. Here the factor $\left(1-u_{n}(t)\right)$ restricts the transition to members of $n_{D H}(t)=\left(1-u_{n}(t)\right) n_{D}(t)$, since in our model only highly frustrated individuals change from non-aggressive to aggressive behaviour. The coefficient $C_{n, H D}$ describes the tendency of $n$ to become more aggressive under hostile 


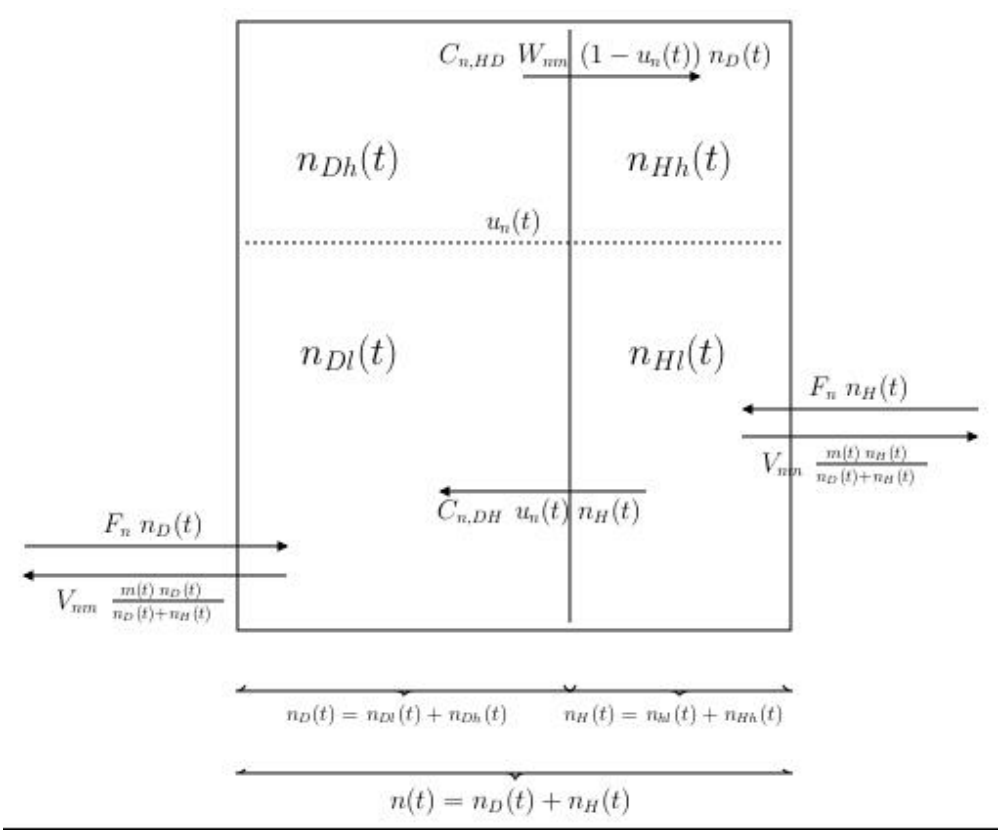

Figure 1: Members of the subpopulation $n_{D}$ which are below $u_{n}(t)$ form the subpopulation $n_{D l}$ (with $l$ for 'low'). Members of the subpopulation $n_{D}$ which are above $u_{n}(t)$ form the subpopulation $n_{D h}$ (with $h$ for 'high'). The same division applies to the subpopulation $n_{H}$.

actions. Thus, $n_{D}$ looses $C_{n, H D} W_{n m} m_{H}\left(1-u_{n}(t)\right) n_{D}(t)$ members and $n_{H}$ gains $C_{n, H D} W_{n m} m_{H}\left(1-u_{n}(t)\right) n_{D}(t)$ members. In total, the number $n(t)$ does not change by this process.

Non-frustrated members of $n_{H}$ belong to $n_{H l}(t)=u_{n}(t) n_{H}(t)$ and these members tend to change from $n_{H}$ to $n_{D}$. The coefficient $C_{n, D H}$ expresses the tendency of $n$ to become more irenic. Thus we have the flux $C_{n, D H} u_{n}(t) n_{H}(t)$ from $n_{H}$ to $n_{D}$. Since we are dealing with an internal flux, the loss for $n_{H}$ is of the same amount but of negative sign as the gain for $n_{D}$. Both internal fluxes are depicted in fig. 1.

The two sources for the two external fluxes considered here are reproduction (birth) and annihilation (dead). The reproduction takes place within $n$, the annihilation of members of $n$ arises from violent action of $m$ against $n$. Let the coefficient $F_{n}$ express the fertility of population $n$. Then $F_{n} n(t)=F_{n}\left(n_{D}(t)+\right.$ $n_{H}(t)$ is the reproduction in this model. We are fully aware that this linear ansatz is a simplification of actual human population growth.

In principle, $m$ could apply several strategies $S_{1}, S_{2}, \ldots$ in the relation to $n$ where $S_{1}, S_{2}, \ldots$ influence the growth rate $d n(t) / d t$ of $n$. The same is true for $n$ with respect to $m$. Actually, we do not model $S_{1}, S_{2}, \ldots$ in detail. We just establish a hostile fraction of $m$, namely $m_{H}(t)$ which causes loss to $d n(t) / d t$ 


$$
\begin{aligned}
\frac{d n_{D}}{d t}= & C_{n, D H} u_{n}(t) n_{H}(t)-C_{n, H D} W_{n m} m_{H}(t)\left(1-u_{n}(t)\right) n_{D}(t)- \\
& V_{n m} \frac{m_{H}(t) n_{D}(t)}{n_{D}(t)+n_{H}(t)}+F_{n} n_{D}(t) \\
\frac{d n_{H}}{d t}= & C_{n, H D} W_{n m} m_{H}(t)\left(1-u_{n}(t)\right) n_{D}(t)-C_{n, D H} u_{n}(t) n_{H}(t)- \\
& V_{n m} \frac{m_{H}(t) n_{H}(t)}{n_{D}+n_{H}(t)}+F_{n} n_{H}(t) \\
\frac{d m_{D}}{d t}= & C_{m, D H} u_{m}(t) m_{H}(t)-C_{m, H D} W_{m n} n_{H}(t)\left(1-u_{m}(t)\right) m_{D}(t)- \\
& V_{m n} \frac{n_{H}(t) m_{D}(t)}{m_{D}(t)+m_{H}(t)}+F_{m} m_{D}(t) \\
\frac{d m_{H}}{d t}= & C_{m, H D} W_{m n} n_{H}(t)\left(1-u_{m}(t)\right) m_{D}(t)-C_{m, D H} u_{m}(t) m_{H}(t)- \\
& V_{m n} \frac{n_{H}(t) m_{H}(t)}{m_{D}+m_{H}(t)}+F_{m} m_{H}(t)
\end{aligned}
$$

according to a term $-V_{n m} \frac{m_{H}(t) n_{D}(t)}{n_{D}(t)+n_{H}(t)}$. The coefficient $V_{n m}$ governs the strength of the annihilating influence of $m$ on $n$, we can call $V_{n m}$ the martial coupling constant. ${ }^{1}$

We are ready to put all internal and external growth rates together in order to describe the growth rates $d n_{D}(t) / d t$ and $d n_{H}(t) / d t$ by two differential equations. Finally, we have similar equations for population $m$. Then

The parameters $u_{n}(t)$ and $u_{m}(t)$ play the most important roles in our model. Up to now we have not specified $u_{n}(t)$ and $u_{m}(t)$ in a quantitative manner. A justified detailed functional form for $u_{n}(t)$ would base on psycho-sociological assumptions on the underlying population $n$ (and likewise for $m$ ). In the following section 3 we give $u_{n}(t)$ and $u_{m}(t)$ simple quantitative expressions.

\section{Calculations}

If one examines a differential equation system like (9) - (11), then one wants to find the solutions $n_{D}(t), n_{H}(t), m_{D}(t), m_{H}(t)$. That is, one looks for analytical expressions for $n_{D}(t), n_{H}(t), m_{D}(t), m_{H}(t)$ which solve $(9)$ - (11). Unfortunately, we were not able to find analytical expressions. Therefore, we have to resort to a numerical examination of (9) - (11) . A further question concerns

\footnotetext{
${ }^{1}$ Because of $V_{n m} \frac{m_{H}(t) n_{D}(t)}{n_{D}(t)+n_{H}(t)}+V_{n m} \frac{m_{H}(t) n_{H}(t)}{n_{D}(t)+n_{H}(t)}=V_{n m} m_{H}$ the impact of $m_{H}$ on $n$ is independent from $n(t)$ in our model. Furthermore, this ansatz assumes that all hawks contribute to hostility, not only the highly frustrated hawks.
} 
the equilibrium points $\mathbf{E}$ (also called stationary points) of (9) - (11). Loosely speaking by aid of an one-dimensional example, at an given $\mathbf{E}$ no change of $n(t)$ occurs, thus $d n(t) / d t=0$ for $n(t)=n_{E}$.

\section{$3.1 \quad u_{n}(t)$ and $u_{m}(t)$}

In this section we explore numerically the behaviour of the differential equation system (9) - (11). Several distinct quantities like the coefficients $C_{n, D H}$, $C_{n, H D}, V_{n m}, F_{n}$ enter into (9) - (11). In order to get numerical results, we must give numbers to the coefficients. Unfortunately, this task is difficult to accomplish in an empirical founded manner. All occurring coefficients should be measurable quantities connected to actual features of the populations under investigation. Presently, we are not able to come up with empirical values. Thus, below we choose fairly arbitrary values where we mainly focus on the quantitative proportion among the coefficients. However, for $n$ we had a quite content and irenic population in mind, whereas we imagined $m$ to be more frustrated. See the table 1 for concrete values.

Table 1: Parameter values for numerical experiments.

\begin{tabular}{llll}
\hline \hline term & value & unit & definition \\
\hline$C_{n, H D}$ & 0.5 & $1 / \mathrm{t}$ & transition rate from $D$ to $H$ for $n$ \\
$C_{n, D H}$ & 0.1 & $1 / \mathrm{t}$ & transition rate from $H$ to $D$ for $n$ \\
$C_{m, H D}$ & 0.9 & $1 / \mathrm{t}$ & transition rate from $D$ to $H$ for $m$ \\
$C_{m, D H}$ & 0.05 & $1 / \mathrm{t}$ & transition rate from $H$ to $D$ for $m$ \\
$W_{n m}$ & 0.1 & & sensitivity of $n$ for aggression by $m$ \\
$W_{m n}$ & 0.4 & & sensitivity of $n$ for aggression by $n$ \\
$V_{n m}$ & 0.00005 & $1 / \mathrm{t}$ & efficiency for aggression by $m$ \\
$V_{m n}$ & 0.00005 & $1 / \mathrm{t}$ & efficiency for aggression by $n$ \\
$F_{n}$ & 0.001 & $1 / \mathrm{t}$ & fertility of $n$ \\
$F_{m}$ & 0.002 & $1 / \mathrm{t}$ & fertility of $n$ \\
$n_{D 0}=n_{D}(t=0)$ & 7 & & starting value for $n_{D}$ \\
$n_{H 0}=n_{H}(t=0)$ & 1 & & starting value for $n_{H}$ \\
$m_{D 0}=m_{D}(t=0)$ & 4 & & starting value for $m_{D}$ \\
$m_{H 0}=m_{H}(t=0)$ & 2 & & starting value for $m_{H}$ \\
\hline \hline
\end{tabular}

At first we want to demonstrate the interplay among the populations $n_{D}(t)$, $n_{H}(t), m_{D}(t)$, and $m_{H}(t)$ as function of the parameters $u_{n}(t)$ and $u_{m}(t)$. For this purpose we simply assume sinusoidal functions

$$
u_{n}(t)=\frac{\sin \left(2 \pi \frac{t}{T_{n}}+\phi_{n}\right)+1}{2}
$$




$$
u_{m}(t)=\frac{\sin \left(2 \pi \frac{t}{T_{m}}+\phi_{m}\right)+1}{2}
$$

which reflect in a rough manner the up and down in the development of both $0 \leq u_{n}(t) \leq 1$ and $0 \leq u_{m}(t) \leq 1$. Figure 2 gives the desired impression (but nothing more). As implied by the model, populations $n_{D}$ and $n_{H}$ develop with opposite trends (likewise $m_{D}$ and $m_{H}$ ). Furthermore, the populations $n_{D}$ and $m_{D}$ of type $D$ develop parallel (raise and fall), as likewise the populations $n_{H}$ and $m_{H}$ of type $H$.

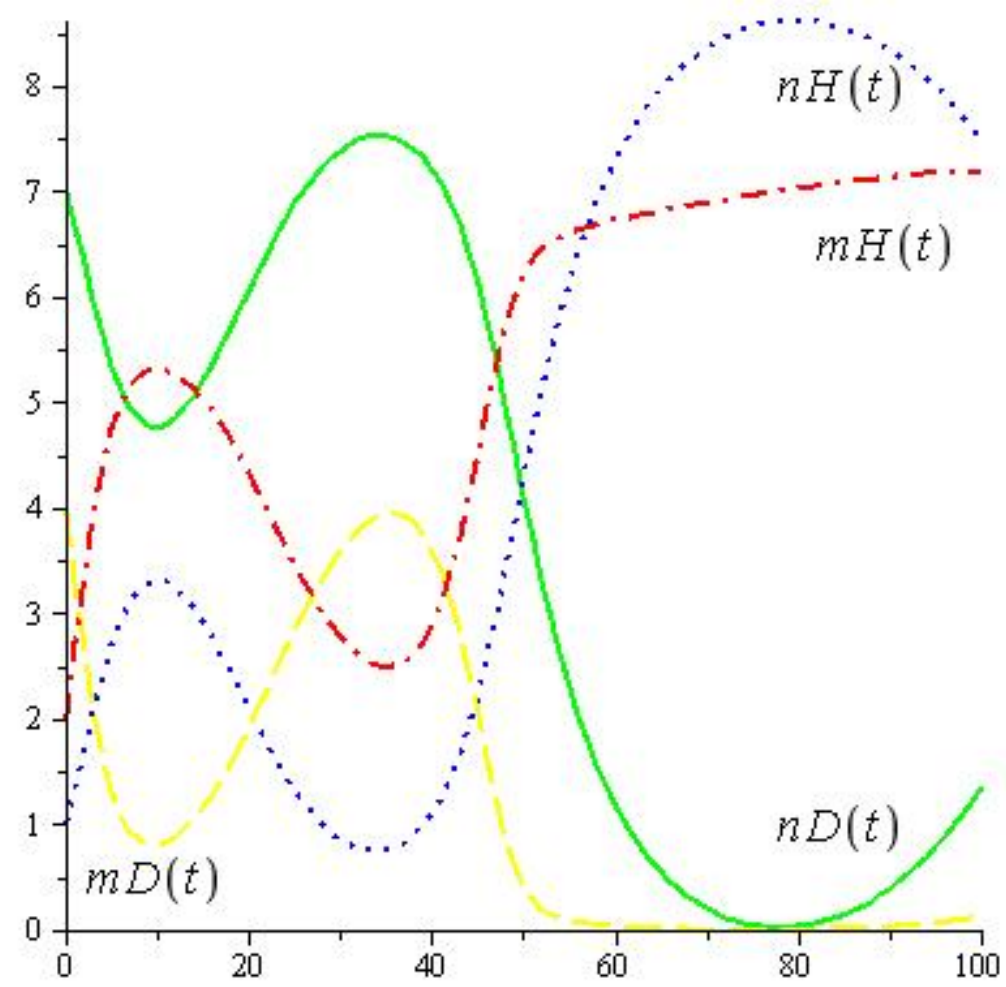

Figure 2: $n_{D}(t)$ (full line), $n_{H}(t)$ (dotted line), $m_{D}(t)$ (dashed line), $m_{H}(t)$ (dashed-dotted line) for $u_{n}(t)$ and $u_{m}(t)$ given by (12) and (13) with $T_{n}=100$, $T_{m}=100, \phi_{n}=0, \phi_{m}=0$.

We draw another qualitative picture if we assume a constantly content population $n$ with $u_{n}(t)=u_{n, 0}=$ const. while the frustration $\left(1-u_{m}(t)\right)$ for population $m$ increases with time according to

$$
\begin{aligned}
u_{n}(t) & =u_{n, 0} \\
u_{m}(t) & =1-\frac{1}{T_{n}} .
\end{aligned}
$$


We are interested in the case where $n$ is of high and constant contentedness $0 \leq u_{n}(t) \leq 1$. From figure (3) we observe that the frustrated and thus aggressive fraction $m_{H}(t)$ increases enormously while the non-frustrated, irenic fraction $m_{D}(t)$ drops down to zero. On the other hand $n_{D}(t)$ remains almost constant over time while $n_{H}(t)$ increases only moderately. Obviously, this situation is far from an equilibrium and explosive.

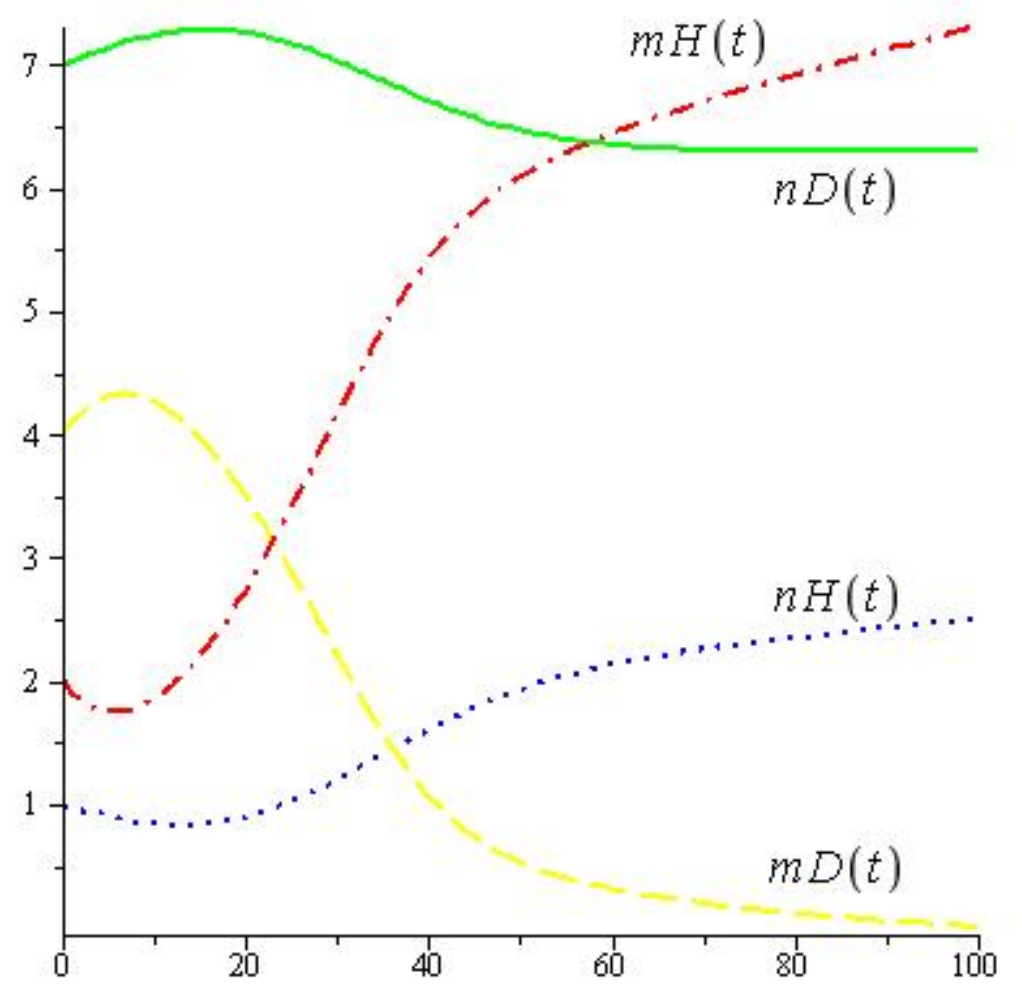

Figure 3: $n_{D}(t)$ (full line), $n_{H}(t)$ (doted line), $m_{D}(t)$ (dashed line), $m_{H}(t)$ (dashed-dotted line) for $u_{n}(t)=u_{n, 0}=0.9$ and $u_{m}(t)=1-\frac{1}{100}$.

\subsection{Equilibrium $\mathrm{E}_{1}$}

Consider the equilibrium points $\mathbf{E}$ of system (9) - (11) for which we have $d n_{D}(t)=d n_{H}(t)=d m_{D}(t)=d m_{H}(t)=0$. In the classical point of view, we will see the subpopulations $n_{D}(t), n_{H}(t), m_{D}(t)$, and $m_{H}(t)$ as the variables by which the equilibrium can be attained. Then the trivial solution for (9) - (11) is $\mathbf{E}_{\mathbf{0}}$ with $n_{D}(t)=n_{H}(t)=m_{D}(t)=m_{H}(t)=0$. However, we also can consider the coefficients which appear in (9) - (11) as variables. This is the point of view which a population $n$ will take if it is confronted by another 
population $m$ and has to somehow change its strategies in order to keep at least $d n(t) / d t=0$ (thus to not parish as for $d n(t) / d t<0$ ).

A first interesting set of parameters is $\left\{V_{n m}, V_{m n}, W_{n m}, W_{m n}\right\}$ because this set expresses the hostile relations among $n(t)$ and $m(t)$ with $V_{n m}=$ the strength of annihilation exerted by $m$ on $n$ and $W_{n m}=$ the strength of the sensitivity ("perception") of $n$ in response to annihilation by $m$. The solution is

$$
\begin{aligned}
V_{m n} & =\frac{\left(m_{D}(t)+m_{H}(t)\right) F_{m}}{n_{H}(t)} \\
V_{n m} & =\frac{\left(n_{D}(t)+n_{H}(t)\right) F_{n}}{m_{H}(t)} \\
W_{m n} & =-\frac{C_{m, D H} u_{m}(t) m_{H}(t)}{\left(u_{m}(t)-1\right) C_{m, H D} n_{H}(t) m_{D}(t)} \\
W_{n m} & =-\frac{C_{n, D H} u_{n}(t) n_{H}(t)}{\left(u_{n}(t)-1\right) C_{n, H D} n_{D}(t) m_{H}(t)}
\end{aligned}
$$

Obviously, the annihilated part $V_{n m} m_{H}(t)$ of $n(t)$ needs to be balanced by $F_{n}\left(n_{D}(t)+n_{H}(t)\right)$ which is the reproduction of $n(t)$. The expression for $W_{n m}$ and $W_{m n}$ can be interpreted similarly as balance of internal flows. It is important to note that $u_{n}(t)$ and $u_{m}(t)$ enter into the solution (16) - (19). Thus, if $u_{n}(t)$ and $u_{m}(t)$ change during time for whatever reason, then $V_{n m}, V_{m n}$, $W_{n m}, W_{m n}$ must be adopted correspondingly in order to stay at the equilibrium for both populations. More dramatically formulated, a population $n$ may be forced to get more aggressive against another population $m$, if both populations interact (as described by this model) and if frustration $\left(1-u_{m}(t)\right)$ increases with time. This can be achieved either by an increase of the coefficients $V_{m n}$ and $W_{n m}$ and/or by an decrease of $u_{n}(t)$.

Yet another set of parameters can lead to $\mathbf{E}_{\mathbf{1}}$, namely $\left\{F_{n}, F_{m}, u_{n}(t), u_{m}(t)\right\}$. Let us recall that $u_{n}(t)$ represents the fraction of content members of $n$ and $(1-u(t))$ is the fraction of frustrated members of $n$ (end likewise for $m$ ). Then $u_{n}(t)$ is an important measure for steering the future of population $n$. Let us have a political leader $\Lambda$ in mind. For example, if $\Lambda$ wants $n$ to become more aggressive against $m$, then $\Lambda$ will manipulate $u_{n}(t)$ to the lowest possible value. On the other hand, if $\Lambda$ is willing to reach a peaceful coexistence of $n$ and $m$, then $\Lambda$ should try to raise $u_{n}(t)$. Thus, in the case of $\left\{F_{n}, F_{m}, u_{n}(t), u_{m}(t)\right\}$ the equilibrium $\mathbf{E}_{1}$ is reached by operating with fertility and with social measures. The resulting expressions for $F_{n}, F_{m}$ are given by $(22)$, (23), see below. For $u_{n}(t), u_{m}(t)$ we have

$$
\begin{aligned}
u_{n} & =\frac{C_{m, H D} W_{m n} n_{H}(t) m_{D}(t)}{C_{m, D H} m_{H}(t)+C_{m, H D} W_{m n} n_{H}(t) m_{D}(t)} \\
u_{m} & =\frac{C_{n, H D} W_{n m} m_{H}(t) n_{D}(t)}{C_{n, D H} n_{H}(t)+C_{n, H D} W_{n m} m_{H}(t) n_{D}(t)}
\end{aligned}
$$




\subsection{Equilibrium $\mathrm{E}_{2}$}

A more natural equilibrium $\mathbf{E}_{\mathbf{2}}$ for (9) - (11) may be seen in $d n_{D}(t)+d n_{H}(t)=$ $0, d m_{D}(t)+d m_{H}(t)=0$. This equilibrium requires that both populations $n$ and $m$ are stable, irrespectively of the internal flows from attitude $D$ to $H$ and vice versa. As a consequence one gets a solution for the parameter set $\left\{V_{n m}, V_{m n}\right\}$ only, $W_{n m}, W_{m n}$ drop out. In this case $\mathbf{E}_{\mathbf{2}}$ is maintained by variable mutual annihilation of $n$ and $m$. The solution is given by equations (16) and (17).

Equilibrium $\mathbf{E}_{\mathbf{2}}$ can also be reached by the parameter set $\left\{F_{n}, F_{m}\right\}$. In this case $\mathbf{E}_{\mathbf{2}}$ is maintained by variable reproduction of $n$ and $m$. In the view of internal and external fluxes in our model system, $\mathbf{E}_{\mathbf{2}}$ thus can be maintained by either by the external fluxes $\left\{V_{n m}, V_{m n}\right\}$ or the external fluxes $\left\{F_{n}, F_{m}\right\}$. In the later case, the reproduction rates then have to fulfill

$$
\begin{aligned}
F_{n} & =\frac{V_{n m} m_{H}(t)}{n_{D}(t)+n_{H}(t)} \\
F_{m} & =\frac{V_{m n} n_{H}(t)}{m_{D}(t)+m_{H}(t)}
\end{aligned}
$$

Although (22) and (23) are algebraically equivalent to (16) and (17) they represent a completely different approach to equilibrium if one thinks of the realization of $\mathbf{E}_{\mathbf{2}}$ in terms of political and social measures.

\subsection{Equilibrium $\mathrm{E}_{3}$}

A third equilibrium $\mathbf{E}_{\mathbf{3}}$ is possible where the internal flows from $n_{D}(t)$ to $n_{H}(t)$ and vice versa cancel each other (likewise for $m$ ). Thus, in case of $\mathbf{E}_{\mathbf{3}}$ one has $d n_{D}(t) / d t=d n_{H}(t) / d t$ and $d m_{D}(t) / d t=d m_{H}(t) / d t . \mathbf{E}_{\mathbf{3}}$ is perhaps the most interesting equilibrium for (9) - (11) since it does not request any growth rate to be equal to zero. Actual populations grow and and it may be desirable to keep an equilibrium for a growing population. For example, it may be desirable to keep the frustrated (and thus aggressive) subpopulation $n_{H}(t)$ as small as possible over time. Solutions for the following sets can be obtained: $\left\{u_{n}(t), u_{m}(t)\right\},\left\{F_{n}(t), F_{m}(t)\right\},\left\{V_{m n}(t), V_{n m}(t)\right\}$ and even $\left\{W_{m n}(t), W_{n m}(t)\right\}$. Unfortunately, the solution expressions are rather long and we omit them here. In any case, $\mathbf{E}_{\mathbf{3}}$ can be attained by proper choice of $\left\{u_{n}(t), u_{m}(t)\right\}$.

\subsection{Non-Equilibria NE}

Observation of real human conflicts shows that a conflict between $n$ and $m$ may persist for (many) generations while both $n$ and $m$ grow in number. Other cases are possible, too. E.g. $n$ may decrease over time and $m$ may increase. In fact, the non-equilibrium states are ubiquitous. As for the equilibria discussed 
in sections $3.2,3.3,3.4$, one can study non-equilibrium states for a given parameter set. Unfortunately, the resulting expressions are much too long to be displayed here.

\section{Discussion}

Sociophysics covers a lot of topics, see for example [1] for a review. The spread and competition of ideas is one example, see [2]. A related topic is the competition of strategies. The present author has tried to capture some features of competing social strategies, see $[5,4,3]$. The main model feature in $[5,4,3]$ are populations which are composed of subpopulations where each subpopulation represents a certain strategy. Populations and subpopulations can grow at the expense of other populations and subpopulations via internal and external interactions among them. Technically these growth processes are modeled by simple differential equations systems.

The present work bases on the same model and extends it by introducing a 'state variable' $u(t)$. This state variable leads to a subdivision of population $n(t)=u_{n}(t) n(t)+\left(1-u_{n}(t)\right) n(t)$ into $u_{n}(t) n(t)=n_{D l}(t)+n_{H l}(t)$ and $\left(1-u_{n}(t)\right) n(t)=n_{D h}(t)+n_{H h}(t)$ where the index $l$ denotes 'low' frustration $\left(1-u_{n}(t)\right)$ and the index $h$ denotes 'high' frustration $\left(1-u_{n}(t)\right)$. Corresponding internal fluxes among the subpopulations $n_{D l}, n_{H l}, n_{D h}, n_{H h}$ are established. Finally, the interaction among the two populations $n$ and $m$ then depends on their internal subpopulations. The aggressive subpopulations $n_{H h}$ and $m_{H h}$ keep on the eternal conflict. We do not show by some intrigue analytical proof that the conflict is eternal. The argument is much more simple: $u(t)$ never stays at $u(t)=0$ but seemingly is beyond control and wanders within $0 \leq u(t) \leq 1$.

Or is $u(t)$ controllable? The answer depends on the model for $u(t)$. Within the present work, designedly no explicit model for $u(t)$ was developed. One main problem in modeling $u(t)$ is the time scale. Political, economical and sociological processes take place on several time scales. Some rapid process interfere with other long-lasting processes. Another problem lies in the relation between the individuals and the society which they constitute. While $u(t)$ is thought as an overall state variable of the population, actually $u(t)$ is carried, suffered and expressed by individuals. However, one motive for the present paper was the (well-known) hypothesis on the connection between frustration and aggressivity. Loosely speaking, frustration caused by poverty, social marginalization and similar social circumstances causes a tendency to aggressivity which then turns against the 'others'.

Is $u(t)$ measurable? Again, the answer depends on the model. However, the present model needs an operational definition of $u(t)$ which allows for a corresponding measurement. Sociology and psychology have their methodological 
apparatus for defining measurable quantities. Admittedly, much work has to be done to turn $u(t)$ into a measurable quantity.

In the meantime, the present model could be developed further. Individuals and societies do not only estimate a situation based on the current absolute values of state variables like $u(t)$ but also on the present change $d u(t) / d t$ in the state variables. Thus, $d u(t) / d t$ and even higher differential may enter into the above differential equation model. Furthermore, the notion of 'population' could be used in more concrete context. A given population may be divided into subpopulations. Tensions among subpopulations are all to well known in the real world.

\section{Conclusion}

Natural sciences like physics are defined by their capability to make predictions based on natural laws. Our model does not allow for a prediction or for forecasting. Is it nevertheless a scientific model? We hope that preliminary models like the one presented here are convertible (in some distant future) into advanced models with prediction capability. The present author does not have the capability for this task.

\section{Appendix}

Some Maple commands which are useful to study the differential equation system (9) - (11).

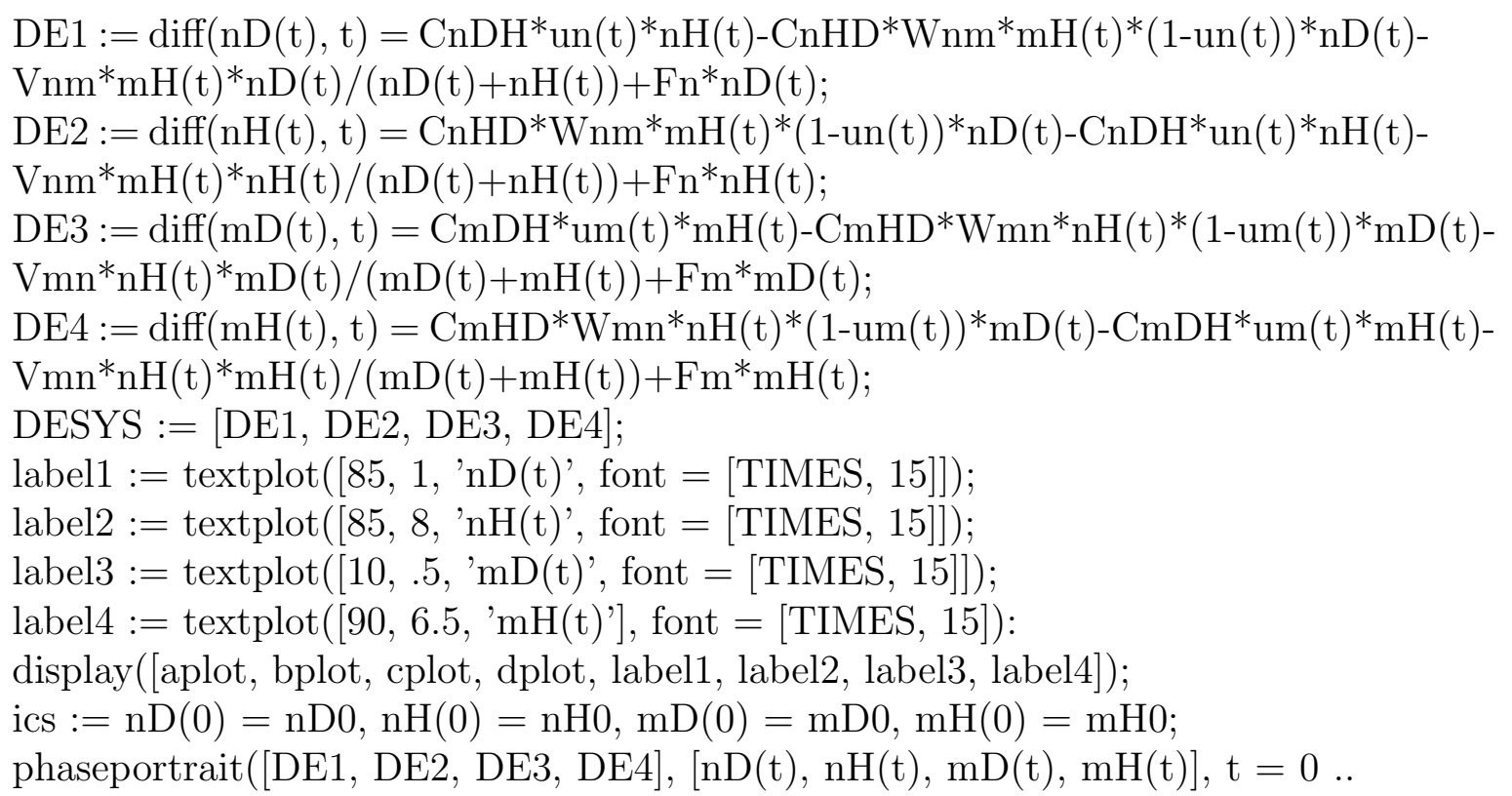


1000, [[ics]],

stepsize $=.1$, scene $=[\mathrm{nD}(\mathrm{t}), \mathrm{nH}(\mathrm{t})]$, linecolour $=\mathrm{t})$;

dsolve('union'(DE1, DE2, DE3, DE4, ics), $\mathrm{mD}(\mathrm{t}), \mathrm{mH}(\mathrm{t}), \mathrm{nD}(\mathrm{t}), \mathrm{nH}(\mathrm{t}))$;

solve $(\operatorname{rhs}(\mathrm{DE} 1)=0, \operatorname{rhs}(\mathrm{DE} 2)=0, \operatorname{rhs}(\mathrm{DE} 3)=0, \operatorname{rhs}(\mathrm{DE} 4)=0, \mathrm{Vmn}, \mathrm{Vnm}$, Wmn, Wnm);

\section{References}

[1] Dietrich Stauffer: A Biased Review of Sociophysics; arXiv:1207.6178 [physics.soc-ph] (2012).

[2] Yubo Wang, Gaoxi Xiao and Jian Liu: Dynamics of competing ideas in complex social systems; New Journal of Physics 14 (2012) 013015 (http://www.njp.org/).

[3] Thomas Wieder: On the use of strategies attached to subpopulations in the description of competition among different populations; International Mathematical Forum, 8(37) (2013), 1839 - 1851. http://dx.doi.org/10.12988/imf.2013.310185

[4] Thomas Wieder: Addendum to 'A simple differential equation system for the description of competition among religions'; International Mathematical Forum 7(54) (2012), 2681 - 2686.

[5] Thomas Wieder: A simple differential equation system for the description of competition among religions; International Mathematical Forum 6(35) (2011), 1713 - 1723.

Received: September 15, 2014; Published: October 28, 2014 